\title{
EXoO-Tn: Tag-n-Map the Tn Antigen in the Human Proteome
}

4 Running title: Tag-n-Map Tn in the Human Proteome

5

6

Weiming Yang*, Minghui Ao, Angellina Song, Yuanwei Xu, and Hui Zhang

11 Department of Pathology, Johns Hopkins University School of Medicine, Baltimore, Maryland,

12 USA.

13

14

$15 *$ Corresponding Author

16 Address: Department of Pathology, Johns Hopkins University School of Medicine, 400 North

17 Broadway, Room 4001A, Baltimore, Maryland, United States.

18 E-mail address: wyang21@jhmi.edu 


\section{Abstract}

20 Tn antigen ( $\mathrm{Tn}$ ), a single $\mathrm{N}$-acetylgalactosamine (GalNAc) monosaccharide attached to protein

21 Ser/Thr residues, is found on most solid tumors yet rarely detected in adult tissues, featuring it

22 one of the most distinctive signatures of cancers. Although it is prevalent in cancers, Tn-

23 glycosylation sites are not entirely clear owing to the lack of suitable technology. Knowing the

24 Tn-glycosylation sites will spur the development of new vaccines, diagnostics, and therapeutics

25 of cancers. Here, we report a novel technology named EXoO-Tn for large-scale mapping of Tn-

26 glycosylation sites. EXoO-Tn utilizes glycosyltransferase C1GalT1 and isotopically-labeled

27 UDP-Gal $\left({ }^{13} \mathrm{C}_{6}\right)$ to tag and convert Tn to Gal $\left({ }^{13} \mathrm{C}_{6}\right)-\mathrm{Tn}$, which has a unique mass being

28 distinguishable to other glycans. This exquisite $\operatorname{Gal}\left({ }^{13} \mathrm{C}_{6}\right)-\mathrm{Tn}$ structure is recognized by

29 OpeRATOR that specifically cleaves N-termini of the Gal $\left({ }^{13} \mathrm{C}_{6}\right)$-Tn-occupied Ser/Thr residues to

30 yield site-containing glycopeptides. The use of EXoO-Tn mapped 947 Tn-glycosylation sites

31 from 480 glycoproteins in Jurkat cells. Given the importance of Tn in diseases, EXoO-Tn is

32 anticipated to have broad utility in clinical studies.

34 Keywords: Tn antigen, site-specific, O-GalNAc, glycoproteomics, cancer 


\section{Introduction}

36 Over decades of biomedical investigations, it was found that one of the most distinctive

37 features of cancers is the expression of $\mathrm{Tn}$ antigen (Tn), which is an N-acetylgalactosamine

38 (GalNAc) attached to protein Ser/Thr residues via an O-linked glycosidic linkage ${ }^{1}$. A variant of

39 Tn is STn, which has an addition of sialic acid monosaccharide ${ }^{1}$. Tn establishes its nature as a

40 pan-carcinoma antigen by finding of its expression in $10-90 \%$ of the solid tumor including lung,

41 prostate, breast, colon, pancreas, gastric, stomach, ovary, cervix, bladder ${ }^{1-3}$. In sharp contrast,

42 the expression of $\mathrm{Tn}$ in adult tissue is rare ${ }^{4}$, making it an attractive target for anti-cancer

43 applications. For instance, Slovin et al. report a Phase I clinical trial using a vaccine consisting

44 of synthetic Tn on a carrier protein for prostate cancer ${ }^{5}$. Studies explore the potential of Tn for

45 early diagnostics ${ }^{6-8}$ and prognostics of cancers ${ }^{9-11}$. To treat cancers, Posey et al. report the

46 development of engineered CAR-T cells that target Tn on mucin protein MUC1 (MUC1-Tn) for

47 killing cancer cells ${ }^{12}$. Also, a Phase I clinical trial using MUC1-Tn specific CAR-T cells started

48 for treating patients with head and neck cancer ${ }^{13,14}$. Despite a noteworthy link between Tn and

49 cancers, the underlying mechanism causing the expression of $\mathrm{Tn}$ in cancers is not entirely clear.

50 It may involve glycosyltransferase C1GalT1 and its chaperone C1GalT1C1 also called Cosmc ${ }^{15}$.

51 Defective mutation in Cosmc is reported to affect the function of C1GalT1 for elongating Tn to

52 normal O-glycan structures ${ }^{15,16}$. Furthermore, Tn is involved in IgA nephropathy (IgAN, also

53 known as Berger's disease) that is the most common glomerular disease in the world ${ }^{3,17,18}$. A

54 large percentage of patients with IgAN progress to kidney failure, also called end-stage renal

55 disease (ESRD) ${ }^{3,17}$. The cause of IgAN may involve the expression of Tn and STn on hinge

56 region of $\operatorname{IgA} 1^{3}$. 
Although Tn is structurally simple, identification of its glycosylation sites and the carrier

58 proteins in the complex samples is highly challenging due to the lack of suitable technology.

59 Limited information regarding Tn-glycosylation sites and carrier proteins hamper the

60 understanding of the role of Tn in cancer biology and the development of new strategies

61 targeting cancers. Current methods for mapping Tn-glycosylation sites include the use of VVA

62 lectin or hydrazide chemistry for the enrichment of Tn-glycopeptides, followed by LC-MS/MS

63 for site localization ${ }^{19,20}$. Jurkat T cells expressing Tn and STn, due to the mutation in Cosmc,

64 are often used as a model system to evaluate the effectiveness of methods. Using VVA lectin

65 chromatography and ETD-MS2, Steentoft et al. identify 68 O-glycoproteins in Jurkat cells ${ }^{19}$.

66 Zheng et al. use galactose oxidase to oxidize Tn followed by solid-phase capture using hydrazide

67 chemistry and release of Tn-glycopeptides using methoxyamine ${ }^{20}$. Subsequent analysis using

68 HCD-MS2 identifies 96 O-glycoproteins in three experiments with 87 glycosylation sites being

69 localized in the first experiment of Jurkat cells ${ }^{20}$. We, however, anticipate that about a thousand

70 Tn-glycosylation sites remain to be mapped in Jurkat cells because 1,295 O-linked glycosylation

71 sites are mapped in CEM cells, a human T cell line, using a method named EXoO developed in

72 previous study ${ }^{21}$. It appears that the development of a technology capable of large-scale

73 mapping of Tn-glycosylation sites would be a significant advance in technology and cancer

74 biology.

75 Here, we introduce a new technology named EXoO-Tn that tags Tn and maps its

76 glycosylation sites in a large-scale. EXoO-Tn utilizes two highly specific enzymes in a one-pot

77 reaction for concurrent tagging of Tn and mapping of its glycosylation sites. The first enzyme is

78 glycosyltransferase C1GalT1, which catalyzes UDP-Gal to add a galactose to Tn. When

79 isotopically-labeled UDP-Gal $\left({ }^{13} \mathrm{C}_{6}\right)$ is used, $\operatorname{Gal}\left({ }^{13} \mathrm{C}_{6}\right)-\mathrm{Tn}$ is formed. The $\operatorname{Gal}\left({ }^{13} \mathrm{C}_{6}\right)-\mathrm{Tn}$ has a 
80 unique mass tag distinguishable to endogenous Gal-GalNAc and other glycans. The second

81 enzyme is an endoprotease named OpeRATOR, which cleaves at N-termini of Ser/Thr residues

82 occupied by the $\operatorname{Gal}\left({ }^{13} \mathrm{C}_{6}\right)$-Tn to release site-containing $\mathrm{Gal}\left({ }^{13} \mathrm{C}_{6}\right)$-Tn-glycopeptides with the

83 glycosylation sites positioning at the N-termini of peptide sequences. The two enzymes are

84 synergistically integrated with the use of solid-phase for optimal removal of contaminants and

85 efficient isolation of site-containing $\mathrm{Gal}\left({ }^{13} \mathrm{C}_{6}\right)$-Tn-glycopeptides. A Proof-of-principle of EXoO-

86 Tn was developed using a synthetic Tn-glycopeptide. The performance of EXoO-Tn was

87 evaluated using Jurkat cells.

88

89 Results

90 Principle of EXoO-Tn

91 EXoO-Tn includes six steps (Fig. 1). (i) Digestion: proteins extracted from samples are digested

92 to peptides. Amino groups on the side chain of Lys residues are modified using guanidination on

93 C18 cartridge. (ii) Enrichment: Tn-glycopeptides are enriched using VVA lectin. (iii)

94 Conjugation: the enriched glycopeptides are conjugated to aldehyde-functionalized solid-phase

95 through amino groups at the peptide N-termini. (iv) Tn-engineering: Tn is catalyzed to $\operatorname{Gal}\left({ }^{13} \mathrm{C}_{6}\right)-$

96 Tn using C1GalT1/C1GalT1C1 and UDP-Gal $\left({ }^{13} \mathrm{C}_{6}\right) . \mathrm{C} 1 \mathrm{GalT} 1 / \mathrm{C} 1 \mathrm{GalT} 1 \mathrm{C} 1$ is specific to modify

97 Tn. The Gal $\left({ }^{13} \mathrm{C}_{6}\right)$-Tn has a unique mass that is distinguishable to endogenous Gal-GalNAc and

98 other glycans in the samples. (v) Release: site-containing Gal $\left({ }^{13} \mathrm{C}_{6}\right)$-Tn-glycopeptides are

99 specifically released from solid-phase using OpeRATOR enzyme, which cleaves N-termini of $100 \mathrm{Gal}\left({ }^{13} \mathrm{C}_{6}\right)$-Tn-occupied Ser/Thr residues. (vi) Analysis: the released glycopeptides are analyzed 101 using LC-MS/MS and software tools. 
102 To show the feasibility of EXoO-Tn, a synthetic Tn-glycopeptide VPSTPPTPS $(\alpha-$

103 GalNAc)PSTPPTPSPSC-NH2 was used (Fig. 2A top left panel). The use of C1GalT1 and UDP-

104 Gal converted Tn to Gal-Tn produced a charge +2 Gal-Tn-glycopeptide at $1149.54 \mathrm{~m} / z$ (Fig. 2A

105 top middle panel), an increase of $\sim 162$ Da corresponding to the mass of a galactose compared to

106 its unmodified counterpart at $1068.51 \mathrm{~m} / \mathrm{z}$ (Fig. 2A top left panel). The Gal-Tn-glycopeptide

107 could be digested by OpeRATOR to yield site-containing glycopeptide S(Gal-

108 Tn)PSTPPTPSPSC-NH2 at $761.34 \mathrm{~m} / \mathrm{z}$ and peptide VPSTPPTP at $795.42 \mathrm{~m} / \mathrm{z}$ (Fig. 2A bottom

109 middle panel). To distinguish the newly engineered Gal-Tn from endogenous Gal-GalNAc and

110 other glycans, the UDP-Gal was substituted by an isotopically-labeled UDP-Gal $\left({ }^{13} \mathrm{C}_{6}\right)$. The

$111 \mathrm{Gal}\left({ }^{13} \mathrm{C}_{6}\right)$ has all six carbon molecules in galactose labeled with carbon-13 featuring an

112 increment mass of $6 \mathrm{Da}$. The use of C1GalT1 and UDP-Gal $\left({ }^{13} \mathrm{C}_{6}\right)$ successfully converted Tn to

113 Gal $\left({ }^{13} \mathrm{C}_{6}\right)$-Tn with a unique mass tag of 371 and yielded a charge +2 Gal $\left({ }^{13} \mathrm{C}_{6}\right)$-Tn-glycopeptide

114 at $1152.55 \mathrm{~m} / \mathrm{z}$ (Fig. 2A top right panel), which had an increase of $\sim 6 \mathrm{Da}$ compared to its charge

$115+2$ Gal-Tn counterpart at $1149.54 \mathrm{~m} / \mathrm{z}$ (Fig. 2A top middle panel). The site-containing

116 glycopeptide $\mathrm{S}\left(\mathrm{Gal}\left({ }^{13} \mathrm{C}_{6}\right)\right.$-Tn)PSTPPTPSPSC-NH2 and peptide VPSTPPTP at 764.35 and 795.42

$117 \mathrm{~m} / \mathrm{z}$, respectively, was generated after OpeRATOR digestion (Fig. 2A bottom right panel). The

118 Gal $\left({ }^{13} \mathrm{C}_{6}\right)$-Tn-glycopeptide had an increase of $\sim 6$ Da compared to its Gal-Tn or endogenous Gal-

119 GalNAc counterpart at $761.34 \mathrm{~m} / \mathrm{z}$ (Fig. 2A bottom middle panel). Next, the MS/MS spectra of

120 site-containing $\operatorname{Gal}\left({ }^{13} \mathrm{C}_{6}\right)$-Tn-glycopeptides were analyzed using HCD-MS2 to identify spectral

121 feature for improvement of confidence of identification. As an illustration, an MS/MS spectrum

122 of site-containing $\operatorname{Gal}\left({ }^{13} \mathrm{C}_{6}\right)$-Tn-glycopeptide from analysis of Jurkat cells was shown (Fig. 2B).

123 A diagnostic oxonium ion generated by HCD fragmentation was observed at $372 \mathrm{~m} / \mathrm{z}$ for the

124 Gal $\left({ }^{13} \mathrm{C}_{6}\right)$-Tn (Fig. 2B). The presence of the diagnostic oxonium ion at $372 \mathrm{~m} / \mathrm{z}$ was utilized in 
125 the data interpretation. The $\operatorname{Gal}\left({ }^{13} \mathrm{C}_{6}\right)$-Tn-glycosylation site was informed to be the Thr residue at

126 the N-terminus of the identified peptide sequence (Fig. 2B). Other fragmentation ions in the

127 MS/MS spectrum, including oxonium ions, peptide b- and y-ions, and peptide ion supported the

128 identification of the glycopeptide (Fig. 2B). The analysis of glycopeptides demonstrated the key

129 enzymatic steps in EXoO-Tn to distinguish Tn from Gal-GalNAc and other glycans by isotopic

130 tagging using C1GalT1 and UDP-Gal $\left({ }^{13} \mathrm{C}_{6}\right)$, and map Tn-glycosylation sites using OpeRATOR

131 and LC-MS/MS.

\section{Mapping site-specific Tn-glycoproteome in Jurkat cells}

134 Jurkat cells were analyzed to evaluate the performance of EXoO-Tn. With 1\% FDR, 3,172

135 peptide-spectrum match (PSM) were assigned to 1,078 unique site-containing $\operatorname{Gal}\left({ }^{13} \mathrm{C}_{6}\right)-\mathrm{Tn}$ -

136 glycopeptides that contained 1,011 unique peptide sequences (Fig. 3 and Supplementary Table

137 1). From the peptide sequence, we mapped $947 \mathrm{Gal}\left({ }^{13} \mathrm{C}_{6}\right)$-Tn-glycosylation sites from 480

138 glycoproteins (Fig. 3 and Supplementary Table 1). The diagnostic oxonium ion at $372 \mathrm{~m} / \mathrm{z}$ was

139 detected in $96.4 \%$ of the assigned MS/MS spectra with an overall intensity being ten-fold lower

140 than that at $204 \mathrm{~m} / \mathrm{z}$ (Fig. 4A and Supplementary Table 1). The detection of oxonium ion at 372

$141 \mathrm{~m} / \mathrm{z}$ in the assigned MS2 spectra supported the presence of $\mathrm{Gal}\left({ }^{13} \mathrm{C}_{6}\right)$ - Tn in the identified

142 glycopeptides (Supplementary Table 1). It was observed that, among the assigned PSMs,

143 approximately $89.2 \%$ glycopeptides were modified by a single $\mathrm{Gal}\left({ }^{13} \mathrm{C}_{6}\right)$ - Tn composition while

144 approximately 9.5 and $1.3 \%$ PSMs were modified by two or three $\operatorname{Gal}\left({ }^{13} \mathrm{C}_{6}\right)$-Tn compositions,

145 respectively (Supplementary Table 1).

147 Characterization of the site-specific Tn-glycoproteome in Jurkat cells 
148 Analysis of the glycosylation sites showed that Thr and Ser accounted for approximately $68.7 \%$

149 and $31.3 \%$, respectively. Motif analysis of \pm 7 amino acids surrounding 946 glycosylation sites

150 found an overrepresentation of Pro residues at the +3 and -1 position (Fig. 4B). Two

151 glycosylation sites residing close to the protein $\mathrm{N}$-termini were not used in the motif analysis.

152 Gene Ontology (GO) analysis of the identified glycoproteins found that integral component of

153 membrane, extracellular exosome, endoplasmic reticulum (ER), Golgi apparatus, cell surface,

154 and extracellular space were enriched for cellular component suggesting the presence of the

155 identified glycoproteins in the secretory pathway and on the cell surface (Fig. 4C). Next, the

156 relative position of the glycosylation sites in protein sequence was plotted and showed that

157 proteins MUC1 and versican core protein (VCAN) had the highest number of glycosylation sites

158 reaching 48 and 11, respectively (Fig. 4D middle panel). Besides, it was observed that the

159 frequency of the glycosylation site was relatively even across protein sequences with lower

160 frequency at protein termini (Fig. 4D top and bottom panels). Comparison of site-specific Tn-

161 glycoproteome identified by EXoO-Tn to two other methods ${ }^{19,20}$ (Supplementary Table 2 and 3)

162 revealed that 888 Tn-glycosylation sites from 398 glycoproteins were exclusively identified

163 using EXoO-Tn (Fig. 4E). Analysis of Jurkat cells established the effectiveness of EXoO-Tn to

164 map the site-specific Tn-glycoproteome in the complex sample.

\section{Discussion}

167 A new technology EXoO-Tn has been developed for large-scale mapping Tn-glycosylation sites

168 in the complex sample. EXoO-Tn has several advantages including (i) large-scale mapping of

169 Tn-glycosylation sites in the complex sample; (ii) a tagging strategy for distinguishing

170 engineered Tn from endogenous Gal-GalNAc and other glycans; (iii) concurrent tagging of Tn 
171 and release of site-containing Tn-glycopeptides from solid-phase in a one-pot fashion; (iv)

172 applicable to analyze mucin-type O-linked glycoproteins; (v) no need of ETD for site

173 localization.

174 C1GalT1 is a natural enzyme with specificity for extending O-GalNAc to core 1 Gal-GalNAc

175 structure. OpeRATOR enzyme is utilized by bacteria to digest mucin glycoproteins in the gut

176 with a specificity at N-termini of Gal-GalNAc occupied Ser/Thr residues. The two enzymes

177 work synergistically to render EXoO-Tn the specificity for mapping Tn-glycosylation sites. It is

178 meritorious that $\mathrm{Tn}$ is tagged to have a unique mass and generate a diagnostic oxonium ion in the

179 MS2 spectrum. The unique mass tag and diagnostic oxonium ion are useful to improve the

180 confidence of identification. The use of solid-phase allows extensive washes that are essential to

181 remove other peptides and contaminants while enables further enrichment of site-containing

182 glycopeptides for LC-MS/MS analysis.

183 We mapped 947 Tn-glycosylation sites from almost 500 glycoproteins, a substantially large

184 number of site-specific Tn-glycoproteome, which demonstrated the effectiveness of EXoO-Tn

185 and supported that a large number of O-linked glycosylation sites could be mapped in cells.

186 Some site-containing Tn-glycopeptides may be too long or too short to be detected using EXoO-

187 Tn with trypsin digestion. Digestion of proteins using proteases with different specificities may

188 further increase the identification number of glycosylation sites in EXoO-Tn methodology. Also,

189 the identification of glycopeptides with two or three $\operatorname{Gal}\left({ }^{13} \mathrm{C}_{6}\right)$-Tn compositions suggests many

190 more glycosylation sites in the peptide sequences supporting an even larger number of Tn-

191 glycosylation sites in Jurkat cells. Characterization of glycosylation sites and glycoproteins

192 identified in Jurkat cells revealed conserved features of protein O-linked glycosylation, including

193 consensus motif, cellular localization, and distribution of the relative position of glycosylation 
194 sites across the protein sequences, a reminiscence of that seen in human kidney, serum, and T

195 cells in the previous study ${ }^{21}$. Given that Tn is prevalent in cancers and other diseases, EXoO-Tn

196 is anticipated to have broad translational and clinical utilities.

\section{Material and Methods}

199 Tagging of Tn and mapping its glycosylation site using synthetic Tn-glycopeptide

200 Synthetic Tn-glycopeptide VPSTPPTPS( $\alpha$-GalNAc)PSTPPTPSPSC-NH2 IgA1 hinge peptide

201 was purchased from Susses Research. In the workflow with sequential enzymatic treatments, five

$202 \mu \mathrm{g}$ of glycopeptide in $50 \mathrm{mM}$ Tris- $\mathrm{HCl} \mathrm{pH} 7.4$ was mixed with one $\mu \mathrm{g}$ recombinant human

203 C1GalT1/C1GalT1C1 protein (R\&D Systems, NM) in the presence of either $0.5 \mathrm{mM}$ UDP-Gal

204 (Sigma-Aldrich) or $0.5 \mathrm{mM} \mathrm{UDP-Gal}{ }^{13} \mathrm{C}_{6}$ (Omicron Biochemicals, lnc., IN) at $37^{\circ} \mathrm{C}$ for 16

205 hours. After incubation, half of each sample was subjected to digestion using five units of

206 OpeRATOR (Genovis Inc, Cambridge, MA) at $37^{\circ} \mathrm{C}$ for 16 hours. The glycopeptides were

207 desalted using C18 ZipTip (Millipore Sigma), dried using speed-vac, and resuspended in $0.1 \%$

208 TFA. In the concurrent one-pot enzymatic treatment that was used in all experiments described

209 below, enzymes including C1GalT1/C1GalT1C1, OpeRATOR, and substrate i.e. UDP-Gal or

210 UDP-Gal ${ }^{13} \mathrm{C}_{6}$ were added at the same time using the amount as described in the above sequential

211 enzymatic workflow and incubated at $37^{\circ} \mathrm{C}$ for 16 hours before $\mathrm{C} 18$ desalting and LC-MS/MS

212 analysis.

\section{Extraction of site-containing Tn-glycopeptides from Jurkat cells}

215 Jurkat Clone E6-1 (NIH AIDS Reagent Program) were cultured and expanded in RPMI 1640

216 supplemented with $10 \%$ fetal bovine serum (FBS), 100 units of penicillin, and $100 \mu \mathrm{g}$ of 
217 streptomycin. The cells were collected, washed three times in the ice-cold PBS and lysed in $8 \mathrm{M}$

218 urea/500 mM ammonia bicarbonate. The cell lyse was sonicated and centrifuged at 16,000 $\mathrm{g}$ to

219 remove particles. Protein concentration was determined using a protein BCA assay. Twenty

220 milligrams of proteins were reduced in $5 \mathrm{mM} \mathrm{DTT}$ at $37^{\circ} \mathrm{C}$ for 1 hour and alkylated in $10 \mathrm{mM}$

221 iodoacetamide at room temperature (RT) for $40 \mathrm{~min}$ in the dark. The samples were then diluted

222 five-fold using $100 \mathrm{mM}$ ammonia bicarbonate buffer. Trypsin was added to the samples with an

223 enzyme/protein ratio of $1 / 40 \mathrm{w} / \mathrm{w}$. After incubation at $37^{\circ} \mathrm{C}$ for 16 hours, lysine residues were

224 guanidination-modified, and peptides were desalted using C18 cartridges (Waters, Milford, MA),

225 as described in the previous study ${ }^{21}$. The peptides were dried using speed-vac, resuspended in

226 PBS with $\alpha 2-3,6,8$ neuraminidase (New England Biolabs, Ipswich, MA), and incubated at $37^{\circ} \mathrm{C}$

227 for 16 hours. Four-hundred microliters agarose bound Vicia Villosa Lectin (VVA) (50\% slurry,

228 Vector Laboratories, Burlingame, CA) were washed twice using water, added to peptides and

229 incubated at RT for 16 hours with rotation. The VVA agarose was gently washed with 1 X PBS

230 for three times. Bound glycopeptides were eluted using $4 \mathrm{M}$ urea/100 mM Tris- $\mathrm{HCl} \mathrm{pH}$

231 7.4/400mM GalNAc (Sigma-Aldrich) at RT for $30 \mathrm{~min}$ with shaking. The eluted glycopeptides

232 were desalted using C18 cartridge and conjugated to AminoLink resin (Pierce, Rockford, IL) as

233 described previously ${ }^{21}$. Briefly, the $\mathrm{pH}$ of $\mathrm{C} 18$ elute containing glycopeptides was neutralized to

234 approximately $\mathrm{pH} 7 \mathrm{using}$ two volume of 10X PBS. The solution was mixed with resin $(100 \mu \mathrm{g}$

235 peptide $/ 100 \mu \mathrm{l}$ resin, $50 \%$ slurry) and $50 \mathrm{mM}$ sodium cyanoborohydride $\left(\mathrm{NaCNBH}_{3}\right)$ at $\mathrm{RT}$ for a

236 minimal of 4 hours or overnight with rotation. Unreacted groups on resin were blocked using $1 \mathrm{M}$

237 Tris- $\mathrm{HCl}$ buffer (pH7.4) with $50 \mathrm{mM} \mathrm{NaCNBH}_{3}$ at RT for $30 \mathrm{~min}$ with rotation. The resin was

238 sequentially washed using 50\% ACN, $1.5 \mathrm{M} \mathrm{NaCl}$, and $50 \mathrm{mM}$ Tris- $\mathrm{HCl}$ buffer (pH 7.4). To tag

239 and release Tn-glycopeptides, a solution $(50 \mu \mathrm{l})$ containing $10 \mu \mathrm{g}$ of C1GalT1/C1GalT1C1, 0.5 
$240 \mathrm{mM}$ UDP-Gal ${ }^{13} \mathrm{C}_{6}$, and 2000 units of OpeRATOR was added to the resin and incubated at $37^{\circ} \mathrm{C}$

241 for 16 hours. The released glycopeptides in the solution were collected twice using $400 \mu 1$ of 50

$242 \mathrm{mM}$ Tris-HCl buffer ( $\mathrm{pH}$ 7.4). Glycopeptides in the collected solution were combined, desalted

243 using C18 cartridge, dried using speed-vac, and resuspended in $0.1 \%$ TFA. The peptides were

244 fractionated using HPLC and concatenated to eight fractions before LC-MS/MS analysis.

\section{LC-MS/MS analysis}

247 One microgram of glycopeptides was analyzed on a Fusion Lumos mass spectrometer with an

248 EASY-nLC 1200 system or an LTQ Orbitrap Velos mass spectrometer (Thermo Fisher

249 Scientific, Bremen, Germany). The mobile phase flow rate was $0.2 \mu \mathrm{l} / \mathrm{min}$ with $0.1 \% \mathrm{FA} / 3 \%$

250 acetonitrile in water (A) and $0.1 \% \mathrm{FA} / 90 \%$ acetonitrile (B). The gradient profile was set as

251 follows: 6\% B for $1 \mathrm{~min}, 6-30 \%$ B for $84 \min , 30-60 \%$ B for $9 \min , 60-90 \%$ B for 1 min, $90 \%$

$252 \mathrm{~B}$ for $5 \mathrm{~min}$ and equilibrated in $50 \% \mathrm{~B}$, flow rate was $0.5 \mu \mathrm{L} / \mathrm{min}$ for $10 \mathrm{~min}$. MS analysis was

253 performed using a spray voltage of $1.8 \mathrm{kV}$. Spectra (AGC target $4 \times 10^{5}$ and maximum injection

254 time $50 \mathrm{~ms}$ ) were collected from 350 to $1800 \mathrm{~m} / \mathrm{z}$ at a resolution of $60 \mathrm{~K}$ followed by data-

255 dependent HCD MS/MS (at a resolution of $50 \mathrm{~K}$, collision energy 36, AGC target of $2 \times 10^{5}$ and

256 maximum IT $250 \mathrm{~ms}$ ) of the 15 most abundant ions using an isolation window of $0.7 \mathrm{~m} / \mathrm{z}$.

257 Include charge state was 2-6. The fixed first mass was $110 \mathrm{~m} / \mathrm{z}$. Dynamic exclusion duration was $25845 \mathrm{~s}$.

\section{Database search of site-containing Tn-glycopeptides}

261 A UniProt human protein database (71,326 entries, downloaded October 19, 2017) was used to 262 generate a peptide database with $26,067,074$ non-redundant peptide entries using the method as 
263 described in the previous study ${ }^{21}$. Briefly, a randomized decoy database using The Trans-

264 Proteomic Pipeline (TPP) ${ }^{22}$ was generated and concatenated with the target database. The

265 concatenated database was digested with trypsin and then OpeRATOR in silico. Peptides with

266 Ser or Thr residues and lengths from 6 to 46 amino acids were used. SEQUEST in Proteome

267 Discoverer 2.2 (Thermo Fisher Scientific) was used to search with variable modification:

268 oxidation (M), $\mathrm{Gal}^{13} \mathrm{C}_{6}(1) \operatorname{HexNAc}(1)(\mathrm{S} / \mathrm{T}), \operatorname{Hex}(1) \operatorname{HexNAc}(1)(\mathrm{S} / \mathrm{T})$ and $\operatorname{HexNAc}(\mathrm{S} / \mathrm{T})$ and

269 static modification: carbamidomethylation (C) and guanidination (K). FDR was set at $1 \%$ using

270 Percolator. Only MS/MS scans with oxonium ion at 204, and two of the other oxonium ions were

271 kept. Assignments with XCorr score below one were removed. MS/MS spectra were manually

272 studied and inspected using spectral viewer in Proteome Discoverer to identify the spectral

273 feature and ensure the confidence of identification.

274

275 Bioinformatics

276 Software pLogo was used to reveal motif for Tn-glycosylation sites ${ }^{23}$ surrounding by 15 amino

277 acids in length with the central amino acids being the sites. The Database for Annotation,

278 Visualization and Integrated Discovery (DAVID) and UniProt (http://www.uniprot.org) were

279 used for Gene Ontology (GO) analysis ${ }^{24}$. Python (version 2.7) is used to analyze the data and

280 generate the figures, including the relative position of Tn-glycosylation sites in protein sequence,

281 radar charts, unsupervised hierarchical clustering, and box plot.

282

\section{Data Availability}

284 The LC-MS/MS data have been deposited to the PRIDE partner repository ${ }^{25}$ with the dataset 285 identifier: project accession: PXD014390 
286 Reviewer account details:

287 Username: reviewer03140@ebi.ac.uk

288 Password: tZVBNHhu

\section{Acknowledgment}

291 We acknowledge Dr. Kyung-Cho Cho for maintenance of Mass Spectrometer. This work was

292 supported by the National Cancer Institute, the Early Detection Research Network (EDRN, 293 U01CA152813), the Clinical Proteomic Tumor Analysis Consortium (CPTAC, U24CA210985), 294 the National Institute of Allergy and Infectious Diseases (R21AI122382), and by amfAR, The 295 Foundation for AIDS Research on Bringing Bioengineers to Cure HIV (Grant amfAR 109551296 61-RGRL). The following reagent was obtained through the NIH AIDS Reagent Program, 297 Division of AIDS, NIAID, NIH: Jurkat Clone E6-1 from Dr. Arthur Weiss (cat\# 177) ${ }^{26}$.

299 Affiliations

300 Department of Pathology, Johns Hopkins University, Baltimore, Maryland, USA.

301 Weiming Yang, Minghui Ao, Angellina Song, Yuanwei Xu, and Hui Zhang 302

\section{Contributions}

304 W.Y. and H.Z. conceived the concept and wrote the manuscript; W.Y., A.S., and Y.X. conducted 305 experiments and data analysis; M.A performed programming, advanced data analysis, and 306 bioinformatics.

\section{Competing financial interests}


bioRxiv preprint doi: https://doi.org/10.1101/840298; this version posted November 13, 2019. The copyright holder for this preprint (which was not certified by peer review) is the author/funder. All rights reserved. No reuse allowed without permission.

309 The authors declare no competing financial interests.

310

\section{Corresponding author}

312 Correspondence to: Weiming Yang

313 


\section{Figures and figure legend}
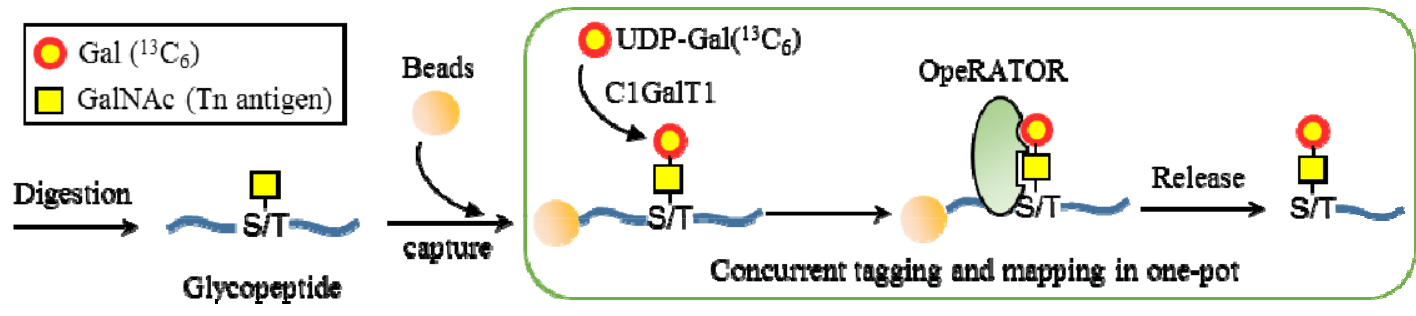

316 Figure 1 Strategy of EXoO-Tn for tagging of Tn and mapping its glycosylation site. 

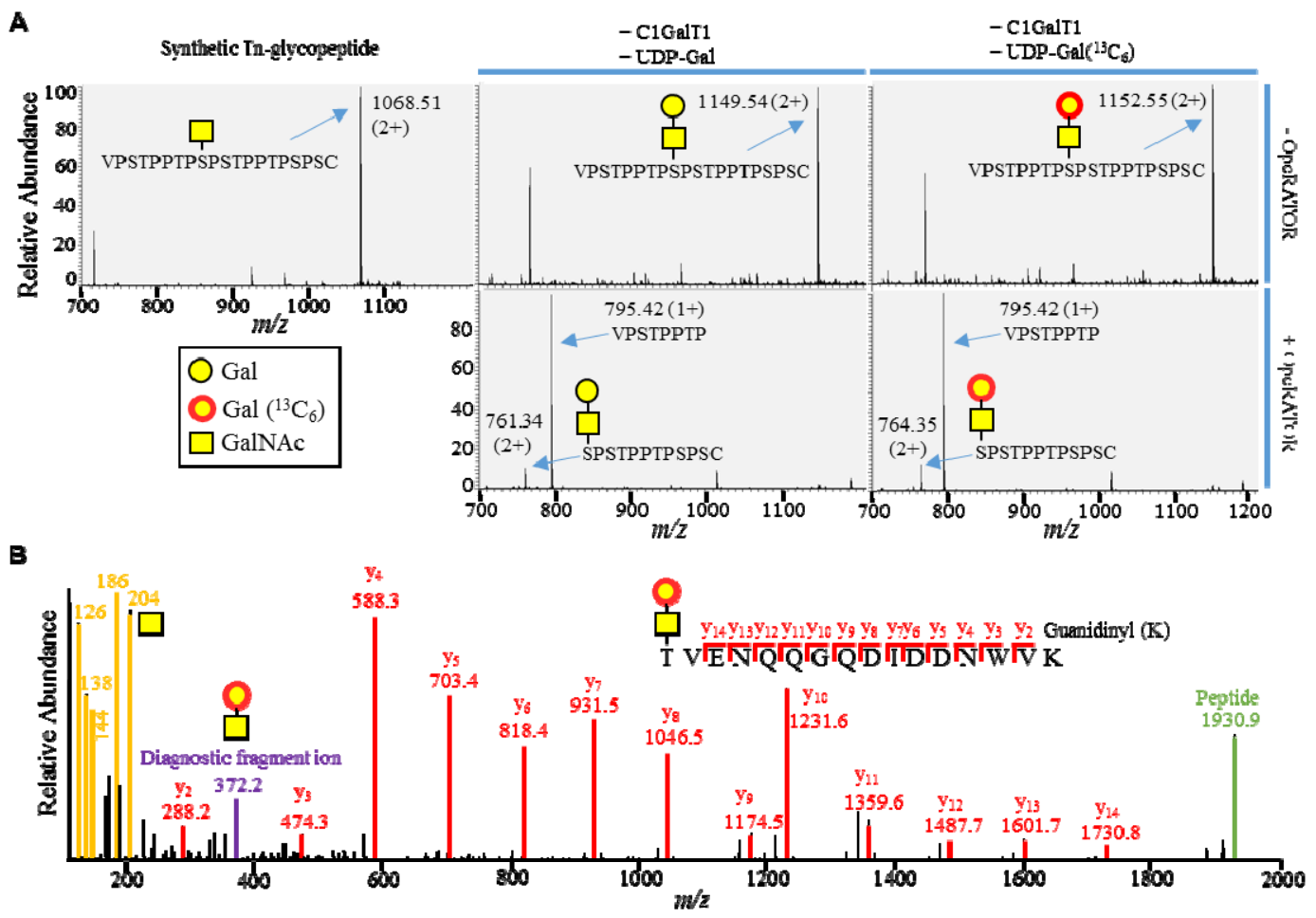

318 Figure 2 Mapping Tn-glycosylation sites by integrating Tn-engineering and OpeRATOR

319 digestion.

320 A. OpeRATOR digestion of Gal- and Gal $\left({ }^{13} \mathrm{C}_{6}\right)$-Tn-glycopeptide after Tn was tagged using

321 C1GalT1 with UDP-Gal or UDP-Gal $\left({ }^{13} \mathrm{C}_{6}\right)$. Top left panel: the synthetic Tn-glycopeptide before

322 treatments. Top middle panel: conversion of Tn to Gal-Tn using C1GalT1 and UDP-Gal. Bottom

323 middle panel: OpeRATOR digestion of the Gal-Tn-glycopeptide generated in the top middle

324 panel produced site-containing glycopeptide S(Gal-Tn)PSTPPTPSPSC-NH2 and peptide

325 VPSTPPTP. Top right panel: conversion of Tn to Gal( $\left.{ }^{13} \mathrm{C}_{6}\right)-\mathrm{Tn}$ using C1GalT1 and UDP-

326 Gal $\left({ }^{13} \mathrm{C}_{6}\right)$. Bottom right panel: OpeRATOR digestion of the $\mathrm{Gal}\left({ }^{13} \mathrm{C}_{6}\right)$-Tn-glycopeptide

327 engineered in the top right panel yielded site-containing glycopeptide $\mathrm{S}\left(\mathrm{Gal}\left({ }^{13} \mathrm{C}_{6}\right)-\right.$

328 Tn)PSTPPTPSPSC-NH2 and peptide VPSTPPTP. B. HCD-MS2 spectrum of site-containing

329 Gal $\left({ }^{13} \mathrm{C}_{6}\right)$-Tn-glycopeptide identified in Jurkat cells. A diagnostic oxonium ion at $372 \mathrm{~m} / \mathrm{z}$

330 corresponding to fragmentation ion of $\mathrm{Gal}\left({ }^{13} \mathrm{C}_{6}\right)$ - Tn was colored in purple. 


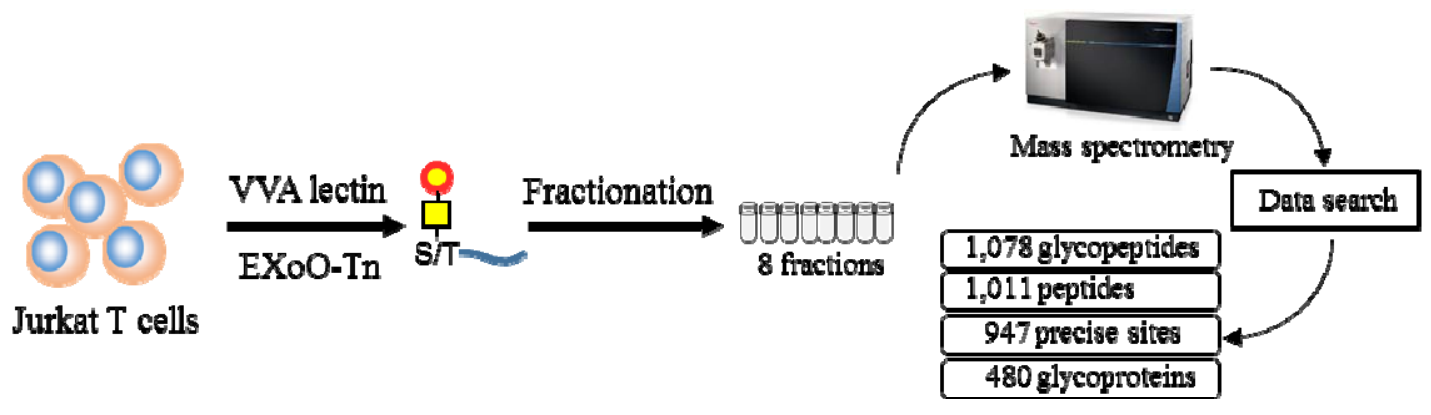

331

332 Figure 3 A Schematic workflow for identification of site-specific Tn-glycoproteome in Jurkat

333 cells.

334 
A

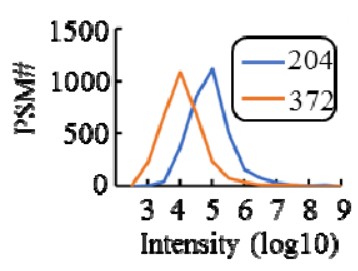

B

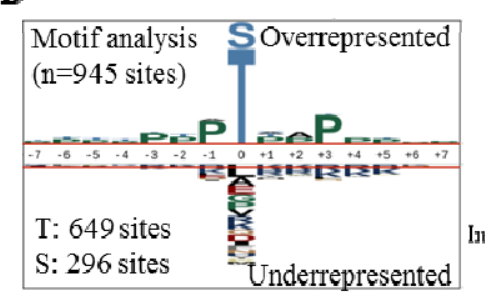

C

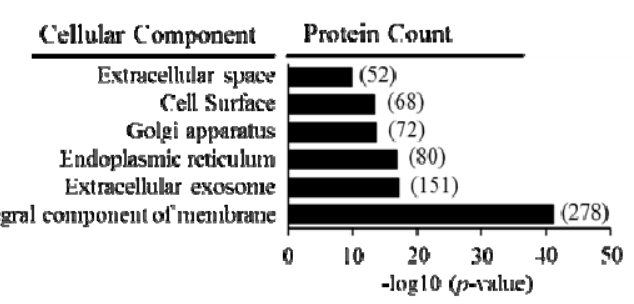

D

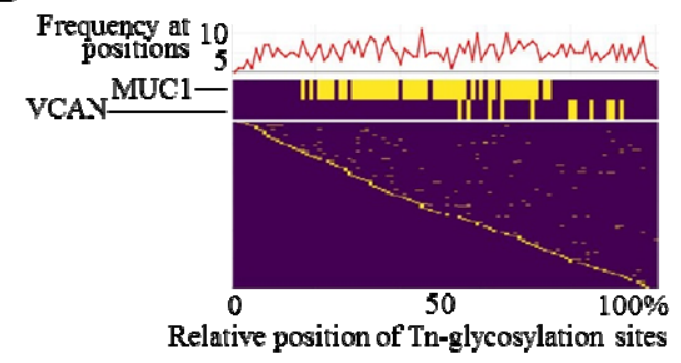

$\mathbf{E}$

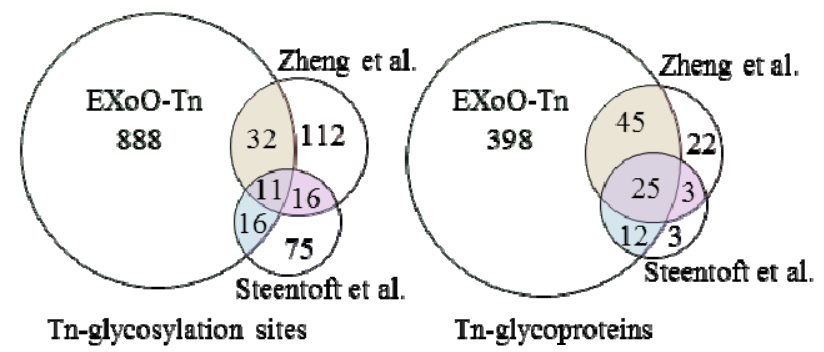

Figure 4 Characteristics of site-specific Tn-glycoproteome in Jurkat cells.

338 A. The overall intensity of oxonium ions at 204 and $372 \mathrm{~m} / \mathrm{z}$ in the assigned PSMs. The overall

339 intensity of oxonium ion at $372 \mathrm{~m} / \mathrm{z}$ was 10 -fold less than that of $204 \mathrm{~m} / \mathrm{z}$. B. Motif analysis

340 revealed the conserved motif of Tn-glycosylation sites. C. GO analysis revealed cellular

341 components for Tn-glycoproteome. D. Analysis of the relative position of Tn-glycosylation sites

342 in protein sequences revealed that the frequency of Tn-glycosylation distributed evenly across

343 protein sequences with lower frequency at protein termini. E. Comparison of O-linked

344 glycosylation sites and glycoproteins identified in this and other studies ${ }^{19,20}$. 


\section{References}

347 1. Julien, S., Videira, P.A. \& Delannoy, P. Sialyl-tn in cancer: (how) did we miss the target? Biomolecules 2, 435-466 (2012).

350

351

352

353

354

355

356

357

358

359

360

361

362

363

364

365

366

367

368

369

370

371

372

373

374

375

376

377

378

379

380

381

382

383

384

385

386

387

388

389

2. Munkley, J. The Role of Sialyl-Tn in Cancer. International journal of molecular sciences 17, 275 (2016).

3. Ju, T. et al. Tn and sialyl-Tn antigens, aberrant 0-glycomics as human disease markers. Proteomics. Clinical applications 7, 618-631 (2013).

4. Kudelka, M.R., Ju, T., Heimburg-Molinaro, J. \& Cummings, R.D. Simple sugars to complex disease--mucin-type 0-glycans in cancer. Advances in cancer research 126, 53-135 (2015).

5. Slovin, S.F. et al. Fully synthetic carbohydrate-based vaccines in biochemically relapsed prostate cancer: clinical trial results with alpha- $\mathrm{N}$-acetylgalactosamine-0serine/threonine conjugate vaccine. Journal of clinical oncology : official journal of the American Society of Clinical Oncology 21, 4292-4298 (2003).

6. Itzkowitz, S.H., Bloom, E.J., Lau, T.S. \& Kim, Y.S. Mucin associated Tn and sialosyl-Tn antigen expression in colorectal polyps. Gut 33, 518-523 (1992).

7. Inoue, M., Ton, S.M., Ogawa, H. \& Tanizawa, O. Expression of Tn and sialyl-Tn antigens in tumor tissues of the ovary. American journal of clinical pathology 96, 711-716 (1991).

8. Wei, H. et al. Glycoprotein screening in colorectal cancer based on differentially expressed Tn antigen. Oncology reports 36, 1313-1324 (2016).

9. Nakagoe, T. et al. Prognostic value of circulating sialyl Tn antigen in colorectal cancer patients. Anticancer research 20, 3863-3869 (2000).

10. Tsuchiya, A. et al. Prognostic Relevance of Tn Expression in Breast Cancer. Breast cancer 6, 175-180 (1999).

11. Ohno, S. et al. Expression of Tn and sialyl-Tn antigens in endometrial cancer: its relationship with tumor-produced cyclooxygenase-2, tumor-infiltrated lymphocytes and patient prognosis. Anticancer research 26, 4047-4053 (2006).

12. Posey, A.D., Jr. et al. Engineered CAR T Cells Targeting the Cancer-Associated TnGlycoform of the Membrane Mucin MUC1 Control Adenocarcinoma. Immunity 44, 1444-1454 (2016).

13. Wilkie, S. et al. Retargeting of human $\mathrm{T}$ cells to tumor-associated MUC1: the evolution of a chimeric antigen receptor. Journal of immunology 180, 4901-4909 (2008).

14. Maher, J. et al. Targeting of Tumor-Associated Glycoforms of MUC1 with CAR T Cells. Immunity 45, 945-946 (2016).

15. Ju, T. et al. Human tumor antigens $\mathrm{Tn}$ and sialyl $\mathrm{Tn}$ arise from mutations in Cosmc. Cancer research 68, 1636-1646 (2008).

16. Hofmann, B.T. et al. COSMC knockdown mediated aberrant 0-glycosylation promotes oncogenic properties in pancreatic cancer. Molecular cancer 14, 109 (2015).

17. Moran, S. \& Cattran, D.C. IgA nephropathy: un update. Minerva medica (2019).

18. Berger, J. \& Hinglais, N. [Intercapillary deposits of IgA-IgG]. Journal d'urologie et de nephrologie 74, 694-695 (1968). 
390 19. Steentoft, C. et al. Mining the 0-glycoproteome using zinc-finger nucleaseglycoengineered SimpleCell lines. Nature methods 8, 977-982 (2011).

20. Zheng, J., Xiao, H. \& Wu, R. Specific Identification of Glycoproteins Bearing the Tn Antigen in Human Cells. Angewandte Chemie 56, 7107-7111 (2017).

21. Yang, W., Ao, M., Hu, Y., Li, Q.K. \& Zhang, H. Mapping the O-glycoproteome using sitespecific extraction of 0-linked glycopeptides (EXoO). Mol Syst Biol 14, e8486 (2018).

22. Deutsch, E.W. et al. Trans-Proteomic Pipeline, a standardized data processing pipeline for large-scale reproducible proteomics informatics. Proteomics. Clinical applications 9, 745-754 (2015).

23. O'Shea, J.P. et al. pLogo: a probabilistic approach to visualizing sequence motifs. Nature methods 10, 1211-1212 (2013).

24. Huang da, W., Sherman, B.T. \& Lempicki, R.A. Systematic and integrative analysis of large gene lists using DAVID bioinformatics resources. Nat Protoc 4, 44-57 (2009).

25. Vizcaino, J.A. et al. 2016 update of the PRIDE database and its related tools. Nucleic acids research 44, D447-456 (2016).

26. Weiss, A., Wiskocil, R.L. \& Stobo, J.D. The role of T3 surface molecules in the activation of human T cells: a two-stimulus requirement for IL 2 production reflects events occurring at a pre-translational level. Journal of immunology 133, 123-128 (1984). 